Le Floch-Prigent P. ${ }^{1}$, Gillot J.-B. ${ }^{2}$, Barbet J.-P. ${ }^{3}$

\title{
Gorilla gorilla: un mannequin anatomique, démontable, grandeur nature du 19ème siècle, en papier mâché de la maison Auzoux, exemplaire non coloré
}

${ }^{1}$ UFR des Sciences de la Santé Paris Simone Veil ; Université de Versailles - Saint Quentin en Yvelines

${ }^{2}$ Librairie Scientifique Alain Brieux, Jean-Bernard GILLOT successeur, 48 rue Jacob, Paris 6ème

${ }^{3}$ Service d'Histologie, Faculté de Médecine, Université Paris Descartes

\begin{abstract}
L'anatomie du Gorille est peu étudiée et publiée, le plus souvent de manière partielle ou incomplète. Dans la deuxième moitié du 19ème siècle, le Docteur Auzoux, fabriquant français de modèles anatomiques en papier mâché qui avait atteint une notoriété internationale, avait reproduit l'anatomie d'un exemplaire de Gorilla gorilla qu'il avait disséqué lui-même. Malgré des spécificités esthétiques : pose accrochée à une branche, représentation en écorché à gauche et pour la partie médiane, représentation essentiellement osseuse à droite, ce modèle était une véritable pièce scientifique car chacun des éléments musculaires, démontables comportait sur sa face non visible outre un numéro d'ordre, l'innervation et l'action, parfois la vascularisation, en en faisant ainsi un véritable recueil de données anatomiques. Cet animal étant en danger d'extinction, ces données quasiment uniques en faisaient un conservatoire morphologique précieux en trois dimensions. Cet exemplaire était non coloré.
\end{abstract}

Keywords: Gorilla gorilla, modèle anatomique, maison Auzoux, papier mâché, 19ème siècle

\section{Patrice Le Floch-Prigent}

LURA 55, Laboratoire d'Anatomie de l' UFR Sciences Médicales Simone Veil, Université de Versailles Saint Quentin, 2, avenue de la source de la Bièvre, 78180 Montigny le Bretonneux e-mail: Patrice.Le-Floch-Prigent@univ-paris5.fr

\section{Introduction}

La maison Brieux possédait en avril 2012 un exemplaire de Gorille grandeur nature, en papier mâché, fabriqué en France par la maison Auzoux, au $19^{\text {ème }}$ siècle et non coloré.

Nous l'avons étudié sur le plan anatomique en prenant en particulier de nombreuses photographies et plusieurs mesures linéaires.

Cet exemplaire malgré son aspect décoratif, accentué par son côté non coloré (le rendant étonnamment conforme aux goûts décoratifs actuels), était un véritable objet scientifique car toutes ses parties démontables étaient non seulement exactes anatomiquement, réalisées d'après dissection d'un exemplaire réel (comme attesté historiquement), mais encore les principales pièces dont les muscles, étaient étiquetés avec leur nom, innervation, action et souvent vascularisation.

Matériel et méthodes

L'examen extérieur montrait un aspect identique sur le plan morphologique aux quelques exemplaires connus, en particulier celui du musée Orfila (fermé définitivement fin 2009) au laboratoire d'anatomie de l'université Paris Descartes, 45 rue des 
Saints Pères, Paris $6^{\text {ème) }}$ étudié par le premier auteur (Le Floch-Prigent, 1995) et qui lui, était colorisé avec conservation éclatante des rouges musculaires sur leurs faces internes qui n'avaient jamais été exposées à la lumière.

La prises de photographies a été réalisée en extérieur (18 avril 2012), en lumière naturelle. Cellesci ont été multipliées sous le plus grand nombre d'incidences possibles, permettant l'étude détaillée, ultérieure des structures anatomiques

Etude bibliographique et du catalogue Auzoux: les références sur l'anatomie macroscopique du Gorille sont éparses. Le catalogue de la maison Auzoux correspondait aux numéros des pièces du spécimen disséqué en 1863.

\section{Résultats}

L'ensemble de la pièce d'un aspect décoratif particulièrement réussi (Figure 1) représentait un gorille femelle (absence de crête crânienne sagittale médiane, et absence d'organes génitaux externes) à taille réelle (hauteur du vertex au socle $=1,57 \mathrm{~m}$, hauteur d'encombrement total de la pièce $=2,20$ $\mathrm{m}$ ), en position bipède avec le membre supérieur droit, osseux en élévation, accroché sur le court prolongement horizontal d'une grosse branche verticale (démontable en deux parties). L'ensemble de la structure était posé sur un socle en bois de $60 \mathrm{~cm}$ de diamètre, par trifurcation inférieure de la structure végétale qui était armée de grosses tiges métalliques, boulonnées sous le socle (avec 4 roulettes probablement modernes).

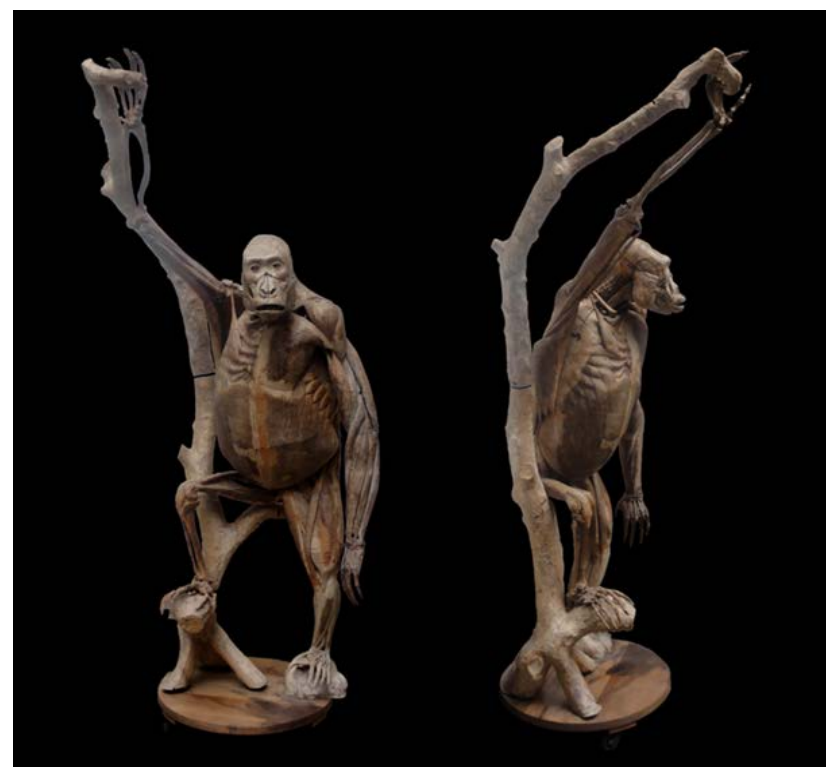

Figure 1 - ensemble de la pièce, vue de face et de troisquart antérieur droit, remarquer l'élément végétal de support apparent (le support principal du gorille anatomique est en réalité ischiatique droit).

Certaines parties du Gorille étaient limitées à l'os (Figure 2: région scapulaire droite) : membre scapulaire droit dont la main était posée sur une branche (le coude était articulé, facilitant par sa flexion et le démontage supérieur de la branche, les manipulations et le déplacement) et membre pelvien droit. Cependant plusieurs muscles étaient représentés au niveau du bras droit, comme également les structures tendineuses du pied droit.

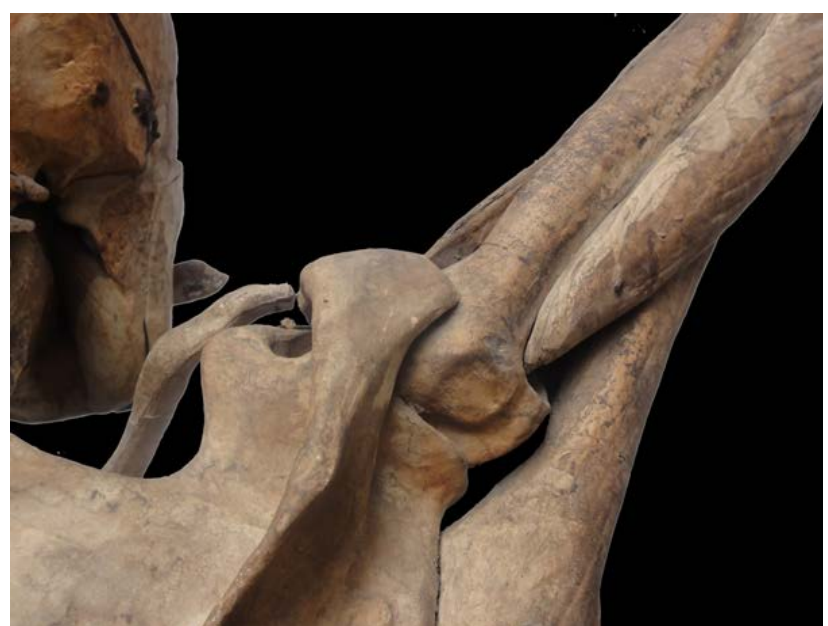

Figure 2 - éléments squelettiques de la région scapulaire, droite. 
La plus grande partie de ce mannequin était constituée d'un écorché musculaire démontable (anatomie clastique du Docteur Auzoux): tête et cou; tronc; et membres scapulaire et pelvien du côté gauche.

Le visage (Figures 3 et 4) était particulièrement expressif avec représentation d'un masque orbiculaire des paupières et les $\mathrm{m}$. zygomatiques.

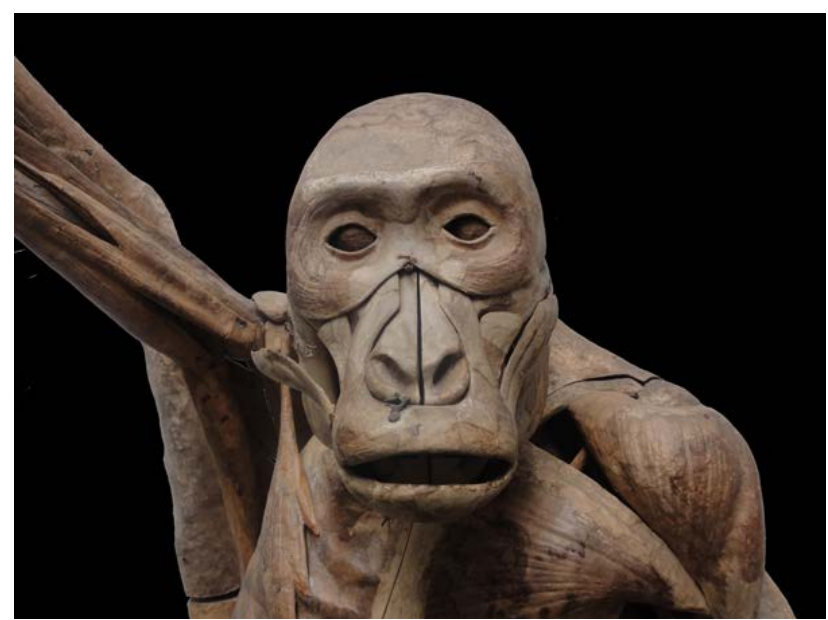

Figure 3 - tête, vue de face

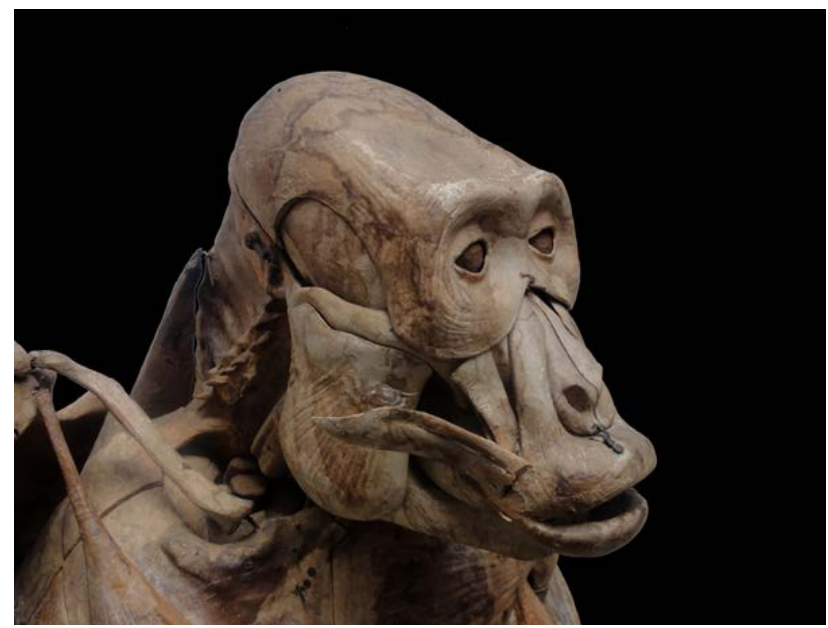

Figure 4 - tête, vue de trois-quart antérieur droit.

Le tronc représentait en superficie les principales masses musculaires avec un rendu très esthétique de l'intrication des insertions costales latérales des $\mathrm{m}$. dentelé antérieur (thoracique) et grand oblique (abdominal)
Le démontage de cet exemplaire non coloré, n'a pas été entrepris d'autant que nombre de ses éléments ont été secondairement cloués les uns sur les autres. L'examen et les photographies s'étaient limités à l'aspect extérieur de la pièce.

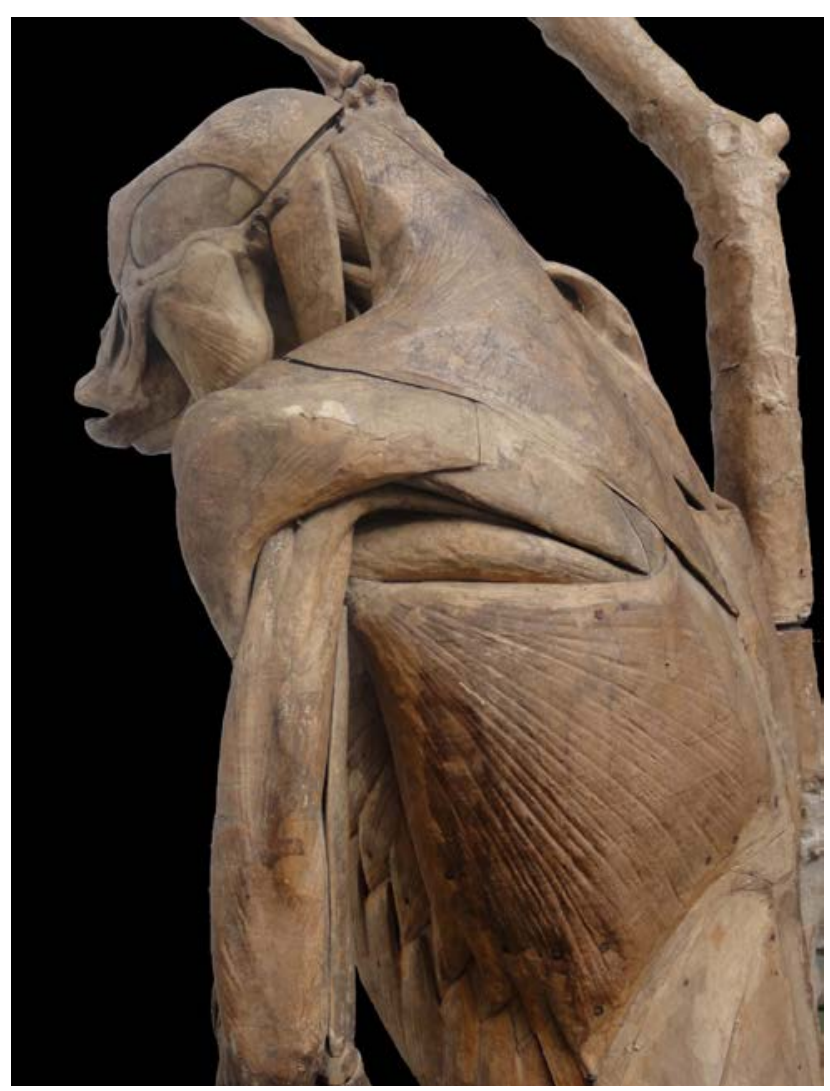

Figure 5 - paroi latérale du tronc, vue de trois-quart arrière gauche.

\section{Discussion}

Il est difficile vu la rareté des éléments zoologiques dissécables de sujets décédés de reproduire des dissections complètes de Gorille. Il est cependant clair que les données historiques concernant cette pièce (dissection par le Dr Auzoux lui-même) 
et les données éparses de la littérature permettent d'affirmer la très grande véracité anatomique de ce modèle d'Auzoux en papier mâché.

Le premier auteur $[1,2]$ avait disséqué dans le Laboratoire d'Anatomie comparée (Pr Jean Anthony) du Musée National d'Histoire Naturelle de Paris, la quasi-totalité d'un Gorille récemment décédé dont plusieurs éléments entiers avaient été scannographies $[3,4,5,6]$.

On peut noter que les pièces «osseuses» de gorille fabriquées en papier mâché, ont été produites en assez grand nombre par la maison Auzoux et sont encore conservées dans les musées d'histoire naturelle (comme le laboratoire d'anatomie comparée du M. N. H. N. de Paris). Outre leur grande véracité anatomique, ces «os » en papier mâché présentent l'intérêt d'une grande légèreté et d'une bonne résistance aux chocs ce qui facilite leur stockage et leur manipulation. L'étude de séries d'os humains, de gorilles et de chimpanzés $[7,8]$ ont permis la découverte de critères non humains [9] au niveau du coude [10] et du pied [11]

L'étude détaillée antérieure [12] avec démontage, photographies extensives et remontage intégral d'un autre exemplaire, identique mais coloré, appartenant au Musée Orfila [13] à Paris, permettait d'affirmer que l'ensemble du mannequin se démontait pièce par pièce jusqu'aux éléments vertébraux, viscères principaux compris. Ce démontage extensif avait permis d'admirer non seulement la véracité anatomique de l'ensemble, l'ingéniosité de la conception et du montage mais aussi la minutie de l'échantillonnage de chaque élément dont les muscles avaient été non seulement numérotés et légendés en caractères d'imprimerie, mais aussi leur action et leur innervation souvent inscrits de la même façon ainsi que parfois leur vascularisation. La lecture du catalogue Auzoux correspondant à cette pièce avec numérotation détaillée de chaque pièce avait été d'autant plus aride que dissociée dans le temps d'avec le démontage de l'ensemble.

\section{Conclusion}

Peu à peu, les études sur les pièces anatomiques en papier mâché prouvent l'intérêt réel de l'ensemble de la production de la maison du Docteur Auzoux qui apparaît sous un autre jour que l'aspect historique et esthétique d'éléments d'une époque qui s'éloigne et paraît désuète.

En réalité, ces mannequins zoologiques, humains comme les pièces botaniques du Docteur Auzoux n'ont pas eu qu'un rôle de diffusion scientifique important, correspondant aux meilleures données de leur époque et n'ont pas été qu'un merveilleux exemple d'ingéniosité et de maîtrise d'un matériau: « le papier mâché » dans la représentation en trois dimensions de pièces morphologiques naturelles. Ce sont aussi et peut-être surtout de véritables pièces scientifiques dont on doit souligner le caractère totalement artificiel alors que la production de cires anatomiques de la maison Tramond [14] sont le plus souvent composites, montées sur des pièces ostéoligamentaires, cadavériques véritables. Tous ces arguments renforcent l'intérêt de leur conservation même pour les exemplaires parfois délaissés de certains départements universitaires d'anatomie ; leur détérioration fréquente mais le plus souvent modérée mérite la restauration.

Le mannequin anatomique du Gorille entier et démontable en papier mâché est un chef d'œuvre inégalé d'anatomie des Primates par sa représentation intégrale des tous les éléments, en particulier osseux et musculaires qui témoignent de la reproduction à l'identique d'une dissection entière et minutieuse d'un vrai Gorille. On peut affirmer que par ce chef d'œuvre probablement inégalable dans le domaine des sciences naturelles, le Docteur Auzoux a laissé un document descriptif et même fonctionnel qui restera à jamais inscrit dans les annales de l'anatomie du Gorille, un des grands Primates proches de l'homme, presque certainement voué à l'extinction rapide dans quelques décennies.

Remerciements: nous remercions l'ensemble de l'équipe de la librairie scientifique Alain Brieux 
: Mes Dara Teste, Caroline Zanon ; ainsi que Madame Brieux et Mr Jimmy Druhlon pour leur aide bibliographique lors de l'étude de l'exemplaire du musée Orfila.

\section{References}

1. Le Floch, P. \& Deloison. Y. (1983). Dissection du pied du chimpanzé et du gorille: comparaisons avec l'homme. $66^{\text {ème }}$ congrès de l'Ass. Anat., Barcelone

2. Le Floch, P. \& Deloison, Y. (1985). Anatomical comparison between entire feet of chimpanzee, gorilla and man (in: TOBIAS, P. éd. - Hominid évolution). New York, Liss, p.135-141

3. Le Floch, P., Jouffroy, F.K. \& Laval-Jeantet, M. (1985). Orientation de l'omoplate et indices thoraciques: scannographie comparée de l'homme, du Gorille et du Chimpanzé. (résumé). Bull Soc. Anat. Paris. 10, 119

4. Le Floch, P., Mandarim de Lacerda, C.A. \& Laval-Jeantet, M. (1985). Scannographie du coude du Gorille: morphologie et biométrie des coupes sériées et comparaisons avec l'homme. (résumé). Bull. Soc. Anat. Paris. 10, 125

5. Le Floch, P. \& Laval-Jeantet, M. (1983). Tomodensitométrie du crâne entier du Gorille dans les 3 plans. Soc. Anat. Paris

6. Le Floch, P. \& Laval-Jeantet, M. (1983). Tomodensitométrie du rachis et de la tête du Gorille et du Chimpanzé : comparaisons avec l'homme. $66^{\text {ème }}$ congrès de l'Ass. Anat., Barcelone

7. Le Floch, P. \& Senut, B. (1981). Sections osseuses sériées fines : approche morphologique, comparative et paléontologique. Exemple de l'extrémité distale chez les primates hominoïdes. C. R. Acad. Sci. Paris. 292(III), 157-162

8. Senut, B. \& Le Floch, P. (1981). Divergence des piliers de la palette humérale chez les Primates Hominoïdes. C. R. Acad. Sci. Paris. 292(III), 757-760
9. Chevrel, J.P. (1994, 1996). Anatomie clinique: tome 2: Le tronc; tome 3: Tête et cou. Paris: Springer

10. Le Floch, P. (1988). L'articulation huméroolécranienne latérale (articulatio humero-ulnaris lateralis): critère non hominien des primates hominoïdes: étude squelettique chez Pan, Gorilla, Pongo, Hylobates et Homo. C. R. Acad. Sci. Paris. 307(III), 535-540

11. LeFloch,P.(1988).L'articulation tarsometatarsea I, critère de bipédie des primates hominoïdes: étude squelettique chez Pan, Gorilla, Pongo, Hylobates et Homo. C. R. Acad. Sci. Paris. 307(III), 577-583

12. Le Floch, P. (1992). Un mannequin anatomique de Gorille du Musée Orfila (Maison Auzoux), (résumé). Bull Soc Anat. Paris. 16, 73-74

13. Delmas, A., Delmas, L., Cabanis, E.A., Delmas, V. \& Iba-Zizen, M.-T. (1995). Les collection des Musées Delmas, Orfila et Rouvière. Surgical and Radiological Anatomy. 17(supp. 1)

14. Lemire, M. (1990). Artistes et mortels, Paris, Chabaud 